\title{
Autonomy, forward non-Zenoness and quadratic stability of bimodal higher-order piecewise linear systems
}

\author{
P. Rapisarda ${ }^{1}$ and M.K. Camlibel ${ }^{2}$
}

\begin{abstract}
We introduce bimodal higher-order piecewise linear systems, i.e. the sets of solutions of two $n$-th order linear differential equations with $n \geq 1$, coupled with an inequality constraint defined by a polynomial differential operator acting on the system trajectories. Under suitable assumptions on the characteristic polynomials of the differential equations and the polynomial associated with the inequality constraint, we prove that a solution always exists and is unique given the initial conditions, that no forward Zeno-behavior occurs, and that the trajectories are continuous together with their first $n-1$ derivatives. Moreover, we prove that such systems are quadratically stable, and we provide an algorithm based on polynomial algebra to compute a Lyapunov function.
\end{abstract}

\section{INTRODUCTION}

Classically, system with state-dependent switching dynamics are studied in a state-space framework, see e.g. [2], [7], [?]. In many situations such an approach is justified from physical principles; however, in many others modelling in state-space form is not justifiable or advisable; see [9], [10], [18] for an elaboration of this viewpoint. These considerations motivate the development of a higher-order framework to piecewise linear systems. Each dynamical mode is described by higher-order differential equations, and the inequalities determining which mode is active are in terms of higher-order polynomial differential operators acting on the system trajectories. In this paper we illustrate some preliminary results in such a framework, limiting ourselves to a special class of scalar bimodal systems described by

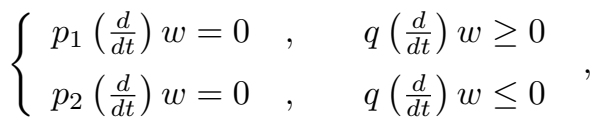

where $p_{i}, q \in \mathbb{R}[\xi]$, and $p_{i}$ is monic, $i=1,2, n:=$ $\operatorname{deg}\left(p_{1}\right)=\operatorname{deg}\left(p_{2}\right)>\operatorname{deg}(q)$, and $\operatorname{GCD}\left(p_{1}, q\right)=1=$ $G C D\left(p_{2}, q\right)$.

We call (1) a (scalar) higher-order linear piecewise system. A trajectory $w: \mathbb{R} \rightarrow \mathbb{R}^{w}$ is called a solution of (1) if it satisfies (1) pointwise over $\mathbb{R}$. We discuss later in this paper the solution space most appropriate to (1), see Prop. 6 and Rem. 7 below. The set of solutions of (1) is the piecewise linear differential behavior (PLDB) associated with (1).

In this paper we prove that if $p_{1}-p_{2}=q$ the system (1) is autonomous (in the sense of [11]) and forward non-Zeno (see [3], [16]). Moreover, we prove that under this assumption, if

\footnotetext{
${ }^{1}$ School of Electronics and Computer Science, University of Southampton, Great Britain, pr3eecs.soton.ac.uk

2 Johann Bernoulli Institute for Mathematics and Computer Science, University of Groningen, The Netherlands, m.k. camlibeldrug.nl
}

$\frac{q}{p_{1}}$ (equivalently, $\frac{q}{p_{2}}$ ) is strictly positive-real then the system (1) admits a quadratic Lyapunov function. We also give a procedure based on polynomial computations to find such a function starting from the description (1)

Following the behavioural approach for switched systems (see [10]), mode equations and inequalities are associated with one-variable polynomials and Lyapunov functionals by two-variable ones. Such theoretical tools lends themselves naturally to modular modelling of complex systems (see [9]) and are conducive to the use of computer algebra techniques. In this paper we strive to provide proofs as self-contained in such framework as possible, or that use arguments based only on elementary mathematical concepts. Whenever possible we outline the relation of our results with those arising in established approaches to systems with state-dependent switching, although the latter are overwhelmingly concerned with first order equations only. Given the space limitations, it is impossible to provide a wider perspective encompassing a more general area, as e.g. in the survey article [4].

The paper is organised as follows: in section II we introduce the basic necessary background concepts. In section III we define and prove the autonomy and forward-nonZenoness of the system (1), and in section IV we prove its quadratic stability. Section V contains an example. In section VI we connect the results presented in this paper with the state-space setting, providing stability results for bimodal piecewise linear systems in state-space form additional to that in [3]. Section VII points out some current research directions.

\section{Notation}

The set of real/complex vectors with an indeterminate but finite number of components is denoted by $\mathbb{R}^{\bullet}$ (respectively $\mathbb{C}^{\bullet}$ ), and the space of $m \times n$ real matrices by $\mathbb{R}^{m \times n}$. The ring of polynomials with real coefficients in the indeterminate $\xi$ is denoted by $\mathbb{R}[\xi]$; the ring of two-variable polynomials with real coefficients in the indeterminates $\zeta$ and $\eta$ is denoted by $\mathbb{R}[\zeta, \eta]$.

The set of infinitely-differentiable (smooth) functions from $\mathbb{R}$ to $\mathbb{R}^{\mathrm{w}}$ is denoted by $\mathfrak{C}^{\infty}\left(\mathbb{R}, \mathbb{R}^{\mathrm{w}}\right)$. A polynomial $p \in \mathbb{R}[\xi]$ is Hurwitz if its roots are all in the open left half-plane. If $f$ is a function defined in a neighbourhood $[t-\epsilon, t)$ of $t \in \mathbb{R}$, we set for $f:[t-\epsilon, t) \rightarrow \mathbb{R}^{\bullet}$ the notation $f\left(t^{-}\right):=$ $\lim _{\tau \nearrow t} f(\tau)$; and similarly for $f:(t, t+\epsilon] \rightarrow \mathbb{R}^{\bullet}$ we set $f\left(t^{+}\right):=\lim _{\tau \searrow t} f(\tau)$, provided that these limits exist. 


\section{PRELIMINARIES}

\section{A. Polynomial differential operators}

In this paper we use the calculus of behaviors as illustrated in [11]. We call $\mathfrak{B} \subseteq \mathfrak{C}^{\infty}\left(\mathbb{R}, \mathbb{R}^{\mathrm{w}}\right)$ a linear time-invariant differential behavior if $\mathfrak{B}$ is the set of solutions of a finite system of constant-coefficient differential equations, i.e., if there exists a polynomial matrix $R \in \mathbb{R}^{\mathrm{g} \times \mathrm{w}}[\xi]$ such that $\mathfrak{B}=$ $\left\{w \in \mathfrak{C}^{\infty}\left(\mathbb{R}, \mathbb{R}^{\mathrm{w}}\right) \mid R\left(\frac{d}{d t}\right) w=0\right\}=\operatorname{ker} R\left(\frac{d}{d t}\right)$. If $\mathfrak{B}$ is represented by $R\left(\frac{d}{d t}\right) w=0$, then we call $R$ a kernel representation of $\mathfrak{B}$. We denote with $\mathfrak{L}^{\mathrm{w}}$ the set of all linear time-invariant differential behaviors with $\mathrm{w}$ variables.

\section{B. Quadratic differential forms}

We use the parametrization of quadratic functionals of system variables and their derivatives using two-variable polynomial matrices introduced in [17].

Let $\Phi \in \mathbb{R}^{\mathrm{w} \times \mathrm{w}}[\zeta, \eta]$; then $\Phi(\zeta, \eta)=\sum_{h, k} \Phi_{h, k} \zeta^{h} \eta^{k}$, where $\Phi_{h, k} \in \mathbb{R}^{\mathrm{w} \times \mathrm{w}}$ and the sum extends over a finite set of nonnegative indices. $\Phi(\zeta, \eta)$ induces the quadratic differential form (QDF) acting on $\mathfrak{C}^{\infty}$-trajectories defined by $Q_{\Phi}(w):=\sum_{h, k}\left(\frac{d^{h} w}{d t^{h}}\right)^{\top} \Phi_{h, k} \frac{d^{k} w}{d t^{k}}$. Since we deal with quadratic forms, we assume without loss of generality that a QDF is induced by a symmetric two-variable polynomial matrix $\Phi(\zeta, \eta)$, i.e. one such that $\Phi(\zeta, \eta)=\Phi(\eta, \zeta)^{\top}$; we denote the set of such matrices by $\mathbb{R}_{s}^{\mathrm{w} \times \mathrm{w}}[\zeta, \eta]$.

Given $Q_{\Psi}$, its derivative is the $\mathrm{QDF} Q_{\Phi}$ defined by $Q_{\Phi}(w):=\frac{d}{d t}\left(Q_{\Psi}(w)\right)$ for all $w \in \mathfrak{C}^{\infty}\left(\mathbb{R}, \mathbb{R}^{\mathrm{w}}\right)$; this holds if and only if $\Phi(\zeta, \eta)=(\zeta+\eta) \Psi(\zeta, \eta)$ (see [17], p. 1710).

$Q_{\Phi}$ is nonnegative along a set of trajectories $\mathfrak{B} \subseteq$ $\mathfrak{C}^{\infty}\left(\mathbb{R}, \mathbb{R}^{\mathrm{w}}\right)$, denoted by $Q_{\Phi} \stackrel{\mathfrak{B}}{\geq} 0$, if $Q_{\Phi}(w) \geq 0$ for all $w \in \mathfrak{B} . Q_{\Phi}$ is positive along $\mathfrak{B}$, denoted by $Q_{\Phi} \stackrel{\mathfrak{B}}{>} 0$, if $Q_{\Phi} \stackrel{\mathfrak{B}}{\geq} 0$ and $\left[Q_{\Phi}(w)=0 \forall w \in \mathfrak{B}\right] \Longrightarrow[w=0]$. If $\mathfrak{B}=\mathfrak{C}^{\infty}\left(\mathbb{R}, \mathbb{R}^{\mathrm{w}}\right)$, then we call $Q_{\Phi}$ simply nonnegative, respectively positive. For algebraic characterizations of these properties see [17], pp. 1712-1713.

\section{Dissipativity}

A controllable (see Ch. 5 of [11]) behaviour $\mathfrak{B} \in \mathfrak{L}^{\mathrm{w}}$ is dissipative with respect to the supply rate $Q_{\Phi}$ (equivalently, $\Phi$-dissipative) if there exists a QDF $Q_{\Psi}$, called a storage function, such that $Q_{\Phi}(w)-\frac{d}{d t} Q_{\Psi}(w) \geq 0$ for all $w \in \mathfrak{B}$. This inequality holds iff there exists a dissipation function, i.e. a $\mathrm{QDF} Q_{\Delta} \stackrel{\mathfrak{B}}{\geq} 0$ such that for all $w \in \mathfrak{B}$ of compact support it holds that $\int_{-\infty}^{+\infty} Q_{\Phi}(w)(t) d t=\int_{-\infty}^{+\infty} Q_{\Delta}(w)(t) d t$ (see Prop. 5.4 of [17]). Moreover, there is a one-one correspondence between storage- and dissipation functions, defined by $\frac{d}{d t} Q_{\Psi}(w)+Q_{\Delta}(w)=Q_{\Phi}(w)$ for all $w \in \mathfrak{B}$. If $\mathfrak{B}=\mathfrak{C}^{\infty}\left(\mathbb{R}, \mathbb{R}^{\mathrm{w}}\right)$, this equality holds if and only if

$$
(\zeta+\eta) \Psi(\zeta, \eta)+\Delta(\zeta, \eta)=\Phi(\zeta, \eta)
$$

Equation (2) is the two-variable polynomial version of the dissipation equality, see section 5 of [17].

Following [1], we call a square matrix of rational functions $B(s)$ positive real if all its entries are analytic in $\mathbb{C}_{+}, B(\lambda)$ is real if $\lambda$ is real, and $B(-\lambda)^{\top}+B(\lambda) \geq 0$ for $\lambda \in$ $\mathbb{C}, \operatorname{Re}(\lambda) \geq 0$. Positive-realness implies that $B(-j \omega)^{\top}+$ $B(j \omega) \geq 0 \forall \omega \in \mathbb{R}$. If the inequality is strict, then $B$ is strictly positive real (note that this term is not universally accepted; cf. e.g. [15], Th. 2.1.). If $\frac{q}{p}$ is strictly positive-real, then it can be shown (see [17]) that the behaviour

$$
\left\{w \in \mathfrak{C}^{\infty}\left(\mathbb{R}, \mathbb{R}^{2}\right) \mid \exists \ell \in \mathfrak{C}^{\infty}(\mathbb{R}, \mathbb{R}) \text { s.t. } w=\left[\begin{array}{c}
p\left(\frac{d}{d t}\right) \\
q\left(\frac{d}{d t}\right)
\end{array}\right] \ell\right\}
$$

is $\left[\begin{array}{ll}0 & 1 \\ 1 & 0\end{array}\right]$-dissipative.

\section{AUTONOMY AND FORWARD NON-ZENONESS}

In this section we examine some issues related to the existence and unicity of solutions to (1); in the classical literature on hybrid and switching systems, these are sometimes conflated in the well-posedness problem (see pp. 44-47 of [4]).

We define an autonomous system (see [11]) as follows.

Definition 1: A set of trajectories $\mathfrak{B}$ is autonomous if for all $\left(w_{1}, w_{2}\right) \in \mathfrak{B}$,

$$
w_{1}(t)=w_{2}(t) \text { for } t<0 \Longrightarrow w_{1}=w_{2} .
$$

In an autonomous set of trajectories the future of every trajectory is completely determined by its past; thus there are no "free" components (inputs) in $w \in \mathfrak{B}$.

Remark 2: In the classical approach to linear complementarity systems (see e.g. [5], [14]), the system dynamics is described by first order differential-algebraic equations:

$$
\begin{aligned}
\frac{d}{d t} x & =f(x, u) \\
0 & =h(x, u) .
\end{aligned}
$$

In that context, a state vector $x_{0} \in \mathbb{R}^{n}$ is called consistent if there exists a solution $(x, u)$ of (3) such that $x(0)=x_{0}$; and autonomous if for every consistent vector $x_{0}$ there exists a unique solution $x(\cdot)$ of (3) such that $x(0)=x_{0}$. Note that such terminology differs also from that used in the hybrid systems community, see p. 34 of [4].

Proposition 3: Consider the dynamics described by (1), and assume that $p_{1}-p_{2}=q$. Then (1) is autonomous.

Proof: The dynamics (1) is equivalently described by

$$
\begin{aligned}
& p_{2}\left(\frac{d}{d t}\right) w=f\left(q\left(\frac{d}{d t}\right) w\right) \\
& f(y):=\left\{\begin{array}{rc}
-y \quad, \quad y \geq 0 \\
0, & y \leq 0
\end{array},\right.
\end{aligned}
$$

It is a matter of straightforward verification to check that $f$ is a Lipschitz function; standard arguments in the theory of differential equations yield that the solution to (4) exists and is unique given the initial conditions; or equivalently, given the trajectory's past. This proves the claim.

We now define forward non-Zenoness (see Remark 3.4.11 p. 55 of [5], and p. 72 of [4]). 
Definition 4: The set of solutions $\mathfrak{B}$ of (1) is forward nonZeno if for all $w \in \mathfrak{B}$ and for all $t^{*} \in \mathbb{R}$, there exist $k \in$ $\{1,2\}$ and $\epsilon\left(w, t^{*}\right)>0$ such that $\left(p_{k}\left(\frac{d}{d t}\right) w\right)(t)=0$ for all $t \in\left(t^{*}, t^{*}+\epsilon\right)$.

Thus in a non-Zeno system only a finite number of mode switches can occur in any finite-length time interval. We now show that under the assumption $p_{1}-p_{2}=q$, the dynamics (1) does not exhibit Zeno behaviour (see also Prop. 6 p. 48 of [4] and Th. 3.2 p. 1933 of [16]).

Proposition 5: Assume that $p_{1}-p_{2}=q$ in the dynamics described by (1). Then the system is forward non-Zeno.

Proof: Define the polynomials $h_{j}^{k}$ by $h_{j}^{k}(s):=$ $s^{j} q(s) \bmod p_{k}(s), j=0, \ldots, n-1, k=1,2$. Note that $\operatorname{deg}\left(h_{j}^{k}\right) \leq n-1, k=1,2, j=0, \ldots, n-1$, and that that if $\left(p_{k}\left(\frac{d}{d t}\right) w\right)(t)=0$, then $\left(h_{j}^{k}\left(\frac{d}{d t}\right) w\right)(t)$ equals the value of the $j$-th derivative of $q\left(\frac{d}{d t}\right) w$ at $t$.

Since $h_{0}^{1}(s)=h_{0}^{2}(s)=q(s)$, it follows that

$$
h_{0}^{1}\left(\frac{d}{d t}\right) w\left(t^{*}\right)=h_{0}^{2}\left(\frac{d}{d t}\right) w\left(t^{*}\right)=q\left(\frac{d}{d t}\right) w\left(t^{*}\right) .
$$

Now if $\left(q\left(\frac{d}{d t}\right) w\right)\left(t^{*}\right)>0$ then there exists a neighbourhood of $t^{*}$ where the solution of $p_{1}\left(\frac{d}{d t}\right) w=0$ is such that $\left(q\left(\frac{d}{d t}\right) w\right)(t)>0$; and if $\left(q\left(\frac{d}{d t}\right) w\right)\left(t^{*}\right)<0$ then a neighbourhood of $t^{*}$ can be found where the solution of $p_{2}\left(\frac{d}{d t}\right) w=0$ is such that $\left(q\left(\frac{d}{d t}\right) w\right)(t)<0$. Thus, the corresponding solution does not exhibit Zeno-behavior.

Now assume that

$h_{0}^{1}\left(\frac{d}{d t}\right) w\left(t^{*}\right)=h_{0}^{2}\left(\frac{d}{d t}\right) w\left(t^{*}\right)=0=q\left(\frac{d}{d t}\right) w\left(t^{*}\right)$

Since $p_{k}$ is monic, $k=1,2$, and writing $q(s)=\sum_{j=0}^{n-1} q_{j} \xi^{j}$, it holds that

$$
\begin{aligned}
h_{1}^{k}(s) & =s q(s) \bmod p_{k}(s) \\
& =s q(s)-q_{n-1} p_{k}(s), k=1,2 .
\end{aligned}
$$

We now prove that $\left(h_{1}^{1}\left(\frac{d}{d t}\right) w\right)\left(t^{*}\right)=\left(h_{1}^{2}\left(\frac{d}{d t}\right) w\right)\left(t^{*}\right)$. If $q_{n-1}=0$, this follows directly from (6). Otherwise, use (6) and subtract $h_{1}^{2}(s)$ from $h_{1}^{1}(s)$ to conclude that since $p_{1}-p_{2}=q$ it also holds that

$$
\begin{aligned}
& h_{1}^{1}\left(\frac{d}{d t}\right) w\left(t^{*}\right)-h_{1}^{2}\left(\frac{d}{d t}\right) w\left(t^{*}\right) \\
& =q_{n-1} p_{1}\left(\frac{d}{d t}\right) w\left(t^{*}\right)-p_{2}\left(\frac{d}{d t}\right) w\left(t^{*}\right) \\
& =q_{n-1} q\left(\frac{d}{d t}\right) w\left(t^{*}\right) .
\end{aligned}
$$

From (5) it follows that

$$
\begin{aligned}
& h_{0}^{k}\left(\frac{d}{d t}\right) w\left(t^{*}\right)=q\left(\frac{d}{d t}\right) w\left(t^{*}\right)=0 \\
& \Longrightarrow h_{1}^{1}\left(\frac{d}{d t}\right) w\left(t^{*}\right)=h_{1}^{2}\left(\frac{d}{d t}\right) w\left(t^{*}\right) .
\end{aligned}
$$

We can follow the argument previously used to prove that there exists a right-neighbourhood of $t^{*}$ where the sign of $q\left(\frac{d}{d t}\right) w$ remains constant, and consequently no Zenobehavior arises. An argument by induction concludes the proof.

We now prove that every solution of (1) is continuous together with its first $n-1$ derivatives.

Proposition 6: Let $w$ be a solution of (1), and assume that $p_{1}-p_{2}=q$; then $\frac{d^{j}}{d t^{j}} w$ is continuous, $j=0, \ldots, n-1$.

Proof: Assume for simplicity of exposition that all roots of $p_{i}$ are distinct, and denote them by $\lambda_{i, k}, i=1,2$, $k=1, \ldots, n:=\operatorname{deg}\left(p_{i}\right)$. Then if $p_{i}\left(\frac{d}{d t}\right)(t)=0$ there exist $\alpha_{i, k}, k=1, \ldots, n$, such that $w(t)=\sum_{k=1}^{n} \alpha_{i, k} e^{\lambda_{i, k} t}$. Consequently, denoting $w^{(j)}:=\frac{d^{j}}{d t^{j}} w$, and defining

$$
E_{i}(t):=\operatorname{diag}\left(e^{\lambda_{i, k} t}\right)_{k=1, \ldots, n}
$$

it holds that

$$
\left[\begin{array}{c}
w(t) \\
\vdots \\
w^{(n)}(t)
\end{array}\right]=\underbrace{\left[\begin{array}{ccc}
1 & \ldots & 1 \\
\lambda_{i, 1} & \ldots & \lambda_{i, n} \\
\vdots & \ldots & \vdots \\
\lambda_{i, 1}^{n-1} & \ldots & \lambda_{i, n}^{n-1} \\
\lambda_{i, 1}^{n} & \ldots & \lambda_{i, n}^{n}
\end{array}\right]}_{=: V_{i}} E_{i}(t) \underbrace{\left[\begin{array}{c}
\alpha_{i, 1} \\
\vdots \\
\alpha_{i, n}
\end{array}\right]}_{\widehat{\alpha}_{i}} .
$$

Now let $t^{*}$ be a switching instant, and assume without loss of generality that $w(t)=\sum_{k=1}^{n} \alpha_{1, k} e^{\lambda_{1, k} t}$ on a neighbourhood of $t^{*}$ (such a neighbourhood exists given the absence of Zeno behaviour, cf. Prop. 5). Denote the coefficient vectors of $q(s), p_{i}(s)=s^{n}+\sum_{k=0}^{n-1} p_{i, k} s^{k}$ with $\widetilde{q}, \widetilde{p}_{i}$, respectively, i.e.

$$
\begin{aligned}
\widetilde{q} & :=\left[\begin{array}{llll}
q_{0} & \ldots & q_{n-1} & 0
\end{array}\right] \\
\widetilde{p_{i}} & :=\left[\begin{array}{llll}
p_{i, 0} & \ldots & p_{i, n-1} & 1
\end{array}\right] .
\end{aligned}
$$

The system switches at $t=t^{*}$ if and only if $\widetilde{q} V_{1} E_{1}\left(t^{*}\right) \widehat{\alpha}_{1}=$ 0 , i.e. if and only if

$$
\widehat{\alpha}_{1} \in\left(\widetilde{q} V_{1} E_{1}\left(t^{*}\right)\right)^{\perp}:=\left\{x \in \mathbb{C}^{n} \mid \widetilde{q} V_{1} E_{1}\left(t^{*}\right) x=0\right\} .
$$

Since $\widetilde{p}_{1}-\widetilde{p}_{2}=\widetilde{q}$ and $\widetilde{p}_{1} V_{1} E_{1}\left(t^{*}\right)=0_{1 \times n}$, it follows that $\left(\widetilde{q} V_{1} E_{1}\left(t^{*}\right)\right)^{\perp} \subseteq\left(\widetilde{p}_{2} V_{1} E_{1}\left(t^{*}\right)\right)^{\perp}$. Following the same argument, it is straightforward to see that also the converse inclusion holds. Moreover, $E_{1}(t)$ is nonsingular for all $t \in \mathbb{R}$, and consequently $\left(\widetilde{p}_{2} V_{1} E_{1}\left(t^{*}\right)\right)^{\perp}=\left(\widetilde{p}_{2} V_{1}\right)^{\perp}$ Now observe that

$$
\widetilde{p}_{2}\left(V_{1}\left(\widetilde{p}_{2} V_{1}\right)^{\perp}\right)=\left(\widetilde{p}_{2} V_{1}\right)\left(\widetilde{p}_{2} V_{1}\right)^{\perp}=\{0\},
$$

and consequently $\widetilde{q} V_{1} E_{1}\left(t^{*}\right) \widehat{\alpha}_{1}$ represents an admissible set of initial conditions for a unique trajectory in ker $p_{2}\left(\frac{d}{d t}\right)$. This implies that the trajectory $w$ and its first $n-1$ derivatives are continuous at $t^{*}$.

The proof of Prop. 6 when one or both of the polynomials $p_{i}$ have repeated roots proceeds analogously, with $E_{i}(\cdot)$ of (7) substituted by a matrix involving terms $t^{j-1} e^{\lambda_{i, k} t}$.

Remark 7: From the result of Prop. 6 it follows that the trajectories of the system (1) are continuous up to their first $n-1$ derivatives. Thus an appropriate choice of the solution space of $(1)$ is $\mathfrak{C}^{n-1}(\mathbb{R}, \mathbb{R})$, the space of $(n-1)$ times differentiable functions. 


\section{QUADRATIC STABILITY}

We now consider the issue of quadratic stability, defined as follows (see pp. 62-70 of [4] for a treatment of Lyapunov stability in the more general context of hybrid systems).

Definition 8: The system (1) is quadratically stable if there exists a quadratic functional $Q_{\Psi}$ of $w$ and its derivatives such that $Q_{\Psi}(w) \geq 0$ and $\frac{d}{d t} Q_{\Psi}(w)<0$ for all nonzero trajectories $w$ of (1).

The main result of this section is the following.

Proposition 9: Assume that $p_{1}-p_{2}=q$ and that $\frac{q}{p_{1}}$ is strictly positive-real. Then the behaviour

$$
\left\{w \in \mathfrak{C}^{\infty}\left(\mathbb{R}, \mathbb{R}^{2} \mid \exists \ell \in \mathfrak{C}^{\infty}(\mathbb{R}, \mathbb{R}) \text { s.t. } w=\left[\begin{array}{c}
p_{1}\left(\frac{d}{d t}\right) \\
q\left(\frac{d}{d t}\right)
\end{array}\right] \ell\right\}\right.
$$

admits a positive storage function $Q_{\Psi}$. Moreover, any such $Q_{\Psi}$ is also a quadratic Lyapunov function for (1). Consequently (1) is quadratically stable.

Proof: Consider first the following result.

Lemma 10: Assume that $p_{1}-p_{2}=q$; then $\frac{q}{p_{1}}$ is strictly positive-real if and only if $\frac{q}{p_{2}}$ is strictly positive-real. Moreover, if $\frac{q}{p_{2}}$ is strictly positive-real then there exists $f \in \mathbb{R}[\xi]$ and $\Psi \in \mathbb{R}[\zeta, \eta]$ such that

$$
p_{2}(\zeta) q(\eta)+q(\zeta) p_{2}(\eta)=(\zeta+\eta) \Psi(\zeta, \eta)+f(\zeta) f(\eta) .
$$

Proof: Assume that $\frac{q}{p_{2}}$ is strictly positive-real, and define $\Phi(\zeta, \eta):=p_{2}(\zeta) q(\eta)+q(\zeta) p_{2}(\eta)$. From the twovariable polynomial version of the dissipation equality (2), it holds that there exist $\Psi \in \mathbb{R}[\zeta, \eta]$ and $f \in \mathbb{R}[\xi]$ such that (9) holds. Moreover $Q_{\Psi} \geq 0$ from Theorem 6.4 of [17]. From the assumption $p_{1}-p_{2}=q$, substituting in (9) and rearranging we obtain

$$
\begin{aligned}
p_{1}(\zeta) q(\eta)+q(\zeta) p_{1}(\eta) & =(\zeta+\eta) \Psi(\zeta, \eta)+f(\zeta) f(\eta) \\
& +2 q(\zeta) q(\eta) .
\end{aligned}
$$

Equation (10) is a dissipation equality for $\frac{q}{p_{1}}$, with storage function being the same as that for $\frac{q}{p_{2}}$, and dissipation rate induced by $2 q(\zeta) q(\eta)+f(\zeta) f(\eta)$. A symmetric argument yields the opposite implication. This yields the equivalence of the strict positive-realness of $\frac{q}{p_{1}}$ and $\frac{q}{p_{2}}$. The rest of the claim follows in a straightforward manner.

We resume the proof of Prop. 9. Let $\Psi$ be any twovariable polynomial as in (9); we prove the claim that $Q_{\Psi}$ is a Lyapunov functional for the dynamics (1).

We first prove that $Q_{\Psi}$ is a Lyapunov function for ker $p_{i}\left(\frac{d}{d t}\right), i=1,2$ in the sense of Th. 4.3 of [17]. It has already been shown in the proof of Lemma 10 that $Q_{\Psi}$ is nonnegative. We now prove that $\frac{d}{d t} Q_{\Psi}$ is strictly negative along nonzero trajectories of ker $p_{i}\left(\frac{d}{d t}\right), i=1,2$. First of all, from the dissipation equality (9) we conclude that $\frac{d}{d t} Q_{\Psi}$ along ker $p_{i}\left(\frac{d}{d t}\right)$ is nonpositive, since it equals $-\left(f\left(\frac{d}{d t}\right) w\right)^{2}$ for (9) and $-\left(f\left(\frac{d}{d t}\right) w\right)^{2}-2\left(q\left(\frac{d}{d t}\right) q\right)^{2}$ for (10). Now assume by contradiction that there exists a trajectory in $w \in \operatorname{ker} p_{2}\left(\frac{d}{d t}\right)$ and $t \in \mathbb{R}$ such that $\frac{d}{d t} Q_{\Psi}(w)(t)=0$. It is straightforward to see that then ker $f\left(\frac{d}{d t}\right)$ and ker $p_{2}\left(\frac{d}{d t}\right)$ have a common trajectory, implying that $f$ and $p_{2}$ have a common root, necessarily in the left-half plane since $p_{2}$ is Hurwitz, being the denominator of a strictly positive-real function (see e.g. [1]). Denote such common root by $\lambda$, and substitute $\zeta=-\lambda$ and $\eta=\lambda$ in (9), obtaining

$$
\underset{=0}{q(-\lambda) p_{2}(\lambda)}+p_{2}(-\lambda) q(\lambda)=f(-\lambda) \underset{=0}{f(\lambda)}=0 ;
$$

from the fact that $p_{2}$ is Hurwitz it follows that $p_{2}(-\lambda) \neq 0$ and consequently $q(\lambda)=0$, contradicting the assumption that $p_{2}$ and $q$ are coprime. An analogous argument proves that $\frac{d}{d t} Q_{\Psi} \stackrel{\text { ker }}{p_{1}\left(\frac{d}{d t}\right)} 0$.

To conclude the proof of the stability of (1), observe that at a switching instant $t^{*}$, because of the continuity of $\frac{d^{j}}{d t^{j}} w, j=0, \ldots, n-1$ established in Prop. 6 it holds that $\lim _{t \rightarrow t^{*}} Q_{\Psi}(w)(t)=\lim _{t \rightarrow t^{*}+} Q_{\Psi}(w)(t)$, and by a standard argument (e.g. that used in the proof of Th. 4.1 of [19]) we conclude that the system is asymptotically stable, and that $Q_{\Psi}$ is a Lyapunov function for (1).

Remark 11: QDFs act on infinitely-differentiable functions, while the trajectories of (1) are not infinitely differentiable; however, this mismatch in differentiability is irrelevant to the result of Prop. 9. Indeed, in the proof we use the calculus of QDFs only as an algebraic tool; moreover, only derivatives up to $n-1$ are involved in the functional equalities corresponding to (9) and (10) (see also Remark 7).

Remark 12: The reader is referred to [8], [9], [10], [12], [13] for results on the relation of positive-realness with the stability of switched systems with state-independent switching.

From the proof of Proposition 9 the following procedure to compute a Lyapunov function can be derived:

\section{Algorithm}

Input: $p_{1}, p_{2}, q \in \mathbb{R}[\xi]$ under the assumptions of Prop. 9

Output: A Lyapunov function for the system (1)

Step 1: Compute $f \in \mathbb{R}[\xi]$ such that

$$
p_{2}(\xi) q(\xi)+q(-\xi) p_{2}(\xi)=f(-\xi) f(\xi)
$$

Comment: $f$ always exists: let $\zeta=-\xi, \eta=\xi$ in (9). $f$ can be computed by spectral factorisation of the LHS of (13).

\section{Step 2: Compute}

$$
\Psi(\zeta, \eta)=\frac{p_{2}(\zeta) q(\eta)+q(\zeta) p_{2}(\eta)-f(\zeta) f(\eta)}{\zeta+\eta}
$$

Step 3: Return $Q_{\Psi}$.

Remark 13: It is possible to translate Steps 1 and 2 of the Algorithm into an equivalent system of linear matrix inequalities involving the coefficients of the polynomials $p_{1}$, $p_{2}$, and the unknown coefficients of $\Psi(\zeta, \eta)$, analogously to what is done in [10]. Such formulation makes it possible to use standard LMI solvers to compute Lyapunov functions for systems like (1). We will not enter into such details here. 


\section{A NUMERICAL EXAMPLE}

Consider the piecewise linear system described by

$$
\begin{aligned}
& \left(\frac{d^{2}}{d t^{2}}+3 \frac{d}{d t}+2\right) w=0 \text { if }\left(\frac{d}{d t}+\frac{1}{2}\right) w \geq 0 \\
& \left(\frac{d^{2}}{d t^{2}}+\frac{3}{2} \frac{d}{d t}+2\right) w=0 \text { if }\left(\frac{d}{d t}+\frac{1}{2}\right) w \leq 0
\end{aligned}
$$

Note that $p_{1}(\xi)=\xi^{2}+3 \xi+2$ and $p_{2}(\xi)=\xi^{2}+\frac{3}{2} \xi+2$ are Hurwitz. Moreover, $q(\xi)=p_{1}(\xi)-p_{2}(\xi)=\xi+\frac{1}{2}$ is such that $\frac{q}{p_{2}}$ and $\frac{q}{p_{1}}$ is strictly positive real. Indeed, $\frac{q}{p_{2}}$ is real, analytic in the closed right half-plane, and moreover

$$
p_{2}(i \omega) q(-i \omega)+p_{2}(-i \omega) q(i \omega)=2\left(1+\omega^{2}\right),
$$

which is positive for all $\omega \in \mathbb{R}$. The positive-realness of $\frac{q}{p_{1}}$ can be proved analogously.

Hence by Proposition 9 we can compute $f(\xi) \in \mathbb{R}[\xi]$ and $\Psi(\zeta, \eta) \in \mathbb{R}[\zeta, \eta]$ such that $Q_{\Psi}$ is a Lyapunov function for $\mathfrak{B}_{1}$ and $\mathfrak{B}_{2}$. It is straightforward to verify that

$$
p_{2}(\xi) q(-\xi)+p_{2}(-\xi) q(\xi)=-2 \xi^{2}+2=f(-\xi) f(\xi) \text {, }
$$

where $f(\xi)=\sqrt{2}(\xi+1)$. The spectral factorisation of the left-hand side of (13) can be performed using standard commands in computer algebra packages.

This choice of $f$ yields the Lyapunov function $Q_{\Psi}$ where

$$
\begin{aligned}
\Psi(\zeta, \eta) & =\frac{p_{2}(\zeta) q(\eta)+q(\zeta) p_{2}(\eta)-f(\zeta) f(\eta)}{\zeta+\eta} \\
& =\frac{1}{4}(3+2 \eta+2 \zeta+4 \zeta \eta) .
\end{aligned}
$$

Applying Proposition 9 we conclude that the linear piecewise system described by (12) is asymptotically stable.

Choosing the alternative spectral factor $\sqrt{2}(\xi-1)$ as $f(\xi)$ in (13) yields a different Lyapunov function, namely that associated with the two-variable polynomial

$$
\Psi^{\prime}(\zeta, \eta):=\frac{1}{4}(4 \eta \zeta+2 \eta+2 \zeta+19) .
$$

\section{CONNECTIONS WITH THE STATE APPROACH}

We now show how Prop. 9 implies a result in the statespace setting, complementary to that illustrated in Th. 3 of sect. 2 of [3]. Assume that $p_{1}, p_{2}$ and $q=p_{1}-p_{2}$ are such that $\frac{q}{p_{1}}$ is strictly positive-real, and write $q(\xi)=q_{0}+\ldots+$ $q_{n-1} \xi^{n-1}$. Let $A_{i} \in \mathbb{R}^{n \times n}$ be the lower companion matrices associated with $p_{i}, i=1,2$, and define $c \in \mathbb{R}^{1 \times n}$ by

$$
c:=\left[\begin{array}{lll}
q_{0} & \ldots & q_{n-1}
\end{array}\right] .
$$

We associate to these matrices the bimodal state-space linear piecewise system described by

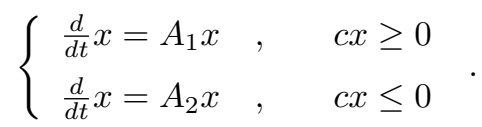

Note that the state variable $x$ of (15) consists of $w$ and its first $n-1$ derivatives:

$$
x=\left[\begin{array}{lll}
w & \ldots & \frac{d^{n-1}}{d t^{n-1}} w
\end{array}\right]^{\top} .
$$

Proposition 14: Assume that $p_{1}-p_{2}=q$, and define $e:=$ $\left[\begin{array}{llll}0 & \ldots & 0 & 1\end{array}\right]^{\top}$. Then $A_{1}-A_{2}=e c$. Moreover $\frac{q(\xi)}{p_{i}(\xi)}=$ $c\left(\xi I-A_{i}\right)^{-1} e, i=1,2$.

If $\frac{q}{p_{1}}$ is strictly positive-real, then there exists $K=K^{\top} \in$ $\mathbb{R}^{n \times n}$ such that

$$
A_{i}^{\top} K+K A_{i} \leq 0, i=1,2 .
$$

Moreover, the dynamics (15) is quadratically stable, and $V(x):=x^{\top} K x$ is a Lyapunov function.

Proof: The proof of the first part of the claim is a matter of straightforward verification and is omitted.

To prove the second part of the Proposition, let $\Psi(\zeta, \eta)$ be as in Prop. 9, and define

$$
S(\xi):=\left[\begin{array}{lll}
1 & \ldots & \xi^{n-1}
\end{array}\right]^{\top} .
$$

It is a matter of straightforward verification to check that

$$
\begin{aligned}
\Psi(\zeta, \eta) & =\sum_{i, j=0}^{n-1} \zeta^{i} \Psi_{i j} \eta^{j} \\
& =S(\zeta)^{\top} \underbrace{\left[\begin{array}{ccc}
\Psi_{00} & \ldots & \Psi_{0, n-1} \\
\vdots & \ldots & \vdots \\
\Psi_{0, n-1} & \ldots & \Psi_{n-1, n-1}
\end{array}\right]}_{=: \widetilde{\Psi}} S(\eta) .
\end{aligned}
$$

Note that since $Q_{\Psi}$ is positive, $\widetilde{\Psi}>0$. We claim that $K \underset{\widetilde{T}}{\text { as }}$ in the statement of Prop. 14 can be chosen to be equal to $\widetilde{\Psi}$.

To prove this, observe that

$$
\xi S(\xi)=A_{i} S(\xi)+e p_{i}(\xi), i=1,2 .
$$

It can be verified equating the coefficients of $\zeta$ and $\eta$ on the left and the right of (9) and (10) that the last row and column of $\widetilde{\Psi}$ contain the coefficient vector of the polynomial $q(\xi)$, and consequently

$$
\begin{aligned}
(\zeta+\eta) \Psi(\zeta, \eta) & =S(\zeta)^{\top}\left(A_{i}^{\top} \widetilde{\Psi}+\widetilde{\Psi} A_{i}\right) S(\eta) \\
& +p_{i}(\zeta) q(\eta)+q(\zeta) p_{i}(\eta)
\end{aligned}
$$

Consequently,

$$
S(\zeta)^{\top}\left(A_{2}^{\top} \widetilde{\Psi}+\widetilde{\Psi} A_{2}\right) S(\eta)=S(\zeta)^{\top} \tilde{f}^{\top} \widetilde{f} S(\eta),
$$

where $\tilde{f}$ is the row-vector consisting of the coefficients of $f(\xi)$. Thus $A_{2}^{\top} \widetilde{\Psi}+\widetilde{\Psi} A_{2}=-\widetilde{f}^{\top} \widetilde{f} \leq 0$.

A similar argument proves that $A_{2}^{\top} \widetilde{\Psi}+\widetilde{\Psi} A_{2}=-\widetilde{h}^{\top} \widetilde{h} \leq 0$, with $\widetilde{h}$ being the coefficient vector of a polynomial $\bar{h}(\xi)$ such that $h(-\xi) h(\xi)=2 q(-\xi) q(\xi)+f(-\xi) f(\xi)$ (that such a polynomial $h$ exists follows from standard spectral factorisation arguments).

To prove that $V(\cdot)$ defined by $V(x)=x^{\top} \widetilde{\Psi} x$ is a Lyapunov function for (1), observe that since $f$ and $p_{2}$ are coprime (see proof of Prop. 9), then $\frac{d}{d t} V(x)<0$ on the trajectories $x(t)=e^{A_{i} t} \bar{x}$, with $\bar{x} \neq 0$ an arbitrary vector in $\mathbb{R}^{n}, i=1,2$. 


\section{CONCLUSIONS}

We have proved autonomy and forward non-Zenoness of solutions of a special class of systems described by two higher-order differential equations and an inequality defined by a polynomial differential operator. Under additional assumptions involving the positive-realness of a rational function derived from the polynomials describing the system, quadratic stability holds. We also provided an algorithm based on standard polynomial algebra to compute a Lyapunov functional.

The results presented here can only be considered to be preliminary to the development of a complete framework to model and analyse higher-order linear complementarity systems using polynomial-algebraic methods. Some issues under study are the establishment of less restrictive sufficient conditions for stability (for example, by relaxing the positivereal assumption), and the extension of our approach to systems described by multivariable differential equations with more than two modes.

\section{ACKNOWLEDGMENT}

P. Rapisarda thanks the Engineering and Physical Sciences Research Council (EPSRC) of Great Britain for financially supporting the visit to the Johann Bernoulli Institute during which the results presented in this paper were worked out (grant EP/L024152/1, "Modeling and Analysis of HigherOrder Switched Linear Systems").

\section{REFERENCES}

[1] B.D.O. Anderson and S. Vongpanitlerd, Network Analysis and Synthesis: A Modern Systems Theory Approach, Prentice-Hall, Inc., NJ, 1973.

[2] S.P. Banks and S.A. Kathur, "Structure and control of piecewise-linear systems" Int. J. Control, vol. 50, no. 2, pp. 667-686, 1989.

[3] Y. Eren, J. Shen and M.K. Çamlibel, "Quadratic stability and stabilization of bimodal piecewise linear systems", Automatica, vol. 50, pp. 1444-1450, 2014.

[4] R. Goebel, R.G. Sanfelice, and A.R. Teel, "Hybrid dynamical systems", IEEE Contr. Syst. Magazine, vol. 29, no. 2, pp. 28-93, 2009.

[5] W.P.M.H. Heemels, Linear Complementarity Systems: A Study in Hybrid Dynamics, Ph.D. Thesis, Technische Universiteit Eindhoven, 1999.

[6] M. Johansson and A. Rantzer, "Computation of piecewise quadratic Lyapunov functions for hybrid systems", IEEE Trans. Aut. Contr., vol. 43, no. 4, pp. 555-559, 1998.

[7] D.M.W. Leenaerts and W.M. van Bokhoven, Piecewise Linear Modeling and Analysis, Kluwer Academic Publishers, Norwell, MA, USA, 1998.

[8] J.C. Mayo-Maldonado and P. Rapisarda, "On positive-realness and stability of switched linear differential systems", Proc. 52nd IEEE $C D C$, Florence, Italy, 2013.

[9] J.C. Mayo-Maldonado and P. Rapisarda, "Modelling of Switching Dynamics in Electrical Systems", Proc. MTNS 2014, Groningen, NL, 2014.

[10] J.C. Mayo-Maldonado, P. Rapisarda and P. Rocha, "Switched linear differential systems and their stability", IEEE Trans. Aut. Contr., vol. 58, no. 8, pp. 2038-2051, 2014.

[11] J.W. Polderman and J.C. Willems, Introduction to Mathematical System Theory: A Behavioral Approach, Springer-Verlag, Berlin,1997.

[12] P. Rapisarda and P. Rocha, "Positive realness and Lyapunov functions for switched behaviors", Proc. MTNS 2012, Melbourne, AU, 2012.

[13] P. Rocha, J.C. Willems, P. Rapisarda, and D. Napp, "On the stability of switched behavioral systems", Proc. 50th IEEE CDC-ECC, Orlando, FL, 2011.
[14] A.J. van der Schaft and J.M. Schumacher, "The complementaryslackness class of hybrid systems", Math. Contr. Sign. Syst., vol. 9, pp. 266-301, 1996.

[15] G. Tao and P.A. Ioannou, "Necessary and sufficient conditions for strictly positive real matrices", Circuits, Devices and Systems, IEE Proceedings G. IET., vol. 137, no. 5, 1990.

[16] L.Q. Thuan and M.K. Çamlibel, "Continuous piecewise affine dynamical systems do not exhibit Zeno behavior", IEEE Trans. Aut. Contr., vol. 56, no. 8, pp. 1932-1936, 2011.

[17] J.C. Willems and H.L. Trentelman, "On quadratic differential forms", SIAM J. Control Optim., vol. 36,pp. 1703-1749, 1998.

[18] J.C. Willems, "The behavioral approach to open and interconnected systems", IEEE Control Systems Magazine, vol. 27, pp. 46-99, 2007.

[19] H. Ye, A.N. Michel, and L. Hou, "Stability theory for hybrid dynamical systems", IEEE Trans. Aut. Contr., vol. 43, no. 4, pp. 461-474, 1998. 